\title{
On orbits of endomorphisms of tori and the Schmidt game
}

\author{
S. G. DANI \\ School of Mathematics, Tata Institute of Fundamental Research, \\ Homi Bhabha Road, Bombay 400005 , India
}

(Received 5 October 1987)

Abstract. We show that there exists a subset $F$ of the $n$-dimensional torus $\mathbb{T}^{n}$ such that $F$ has Hausdorff dimension $n$ and for any $x \in F$ and any semisimple automorphism $\sigma$ of $\mathbb{T}^{n}$ the closure of the $\sigma$-orbit of $x$ contains no periodic points.

\section{Introduction}

Automorphisms and, more generally, surjective endomorphisms of tori are some of the first examples that one learns in ergodic theory. Conditions for the ergodicity of these (with respect to the Haar measure) are well known (cf. [6], for instance) and when ergodicity holds, one knows that the orbits of almost all points are dense. However, not much is known about the exceptional set, of points with non dense orbits, by way of either its location in the torus or its size, in terms other than the measure.

It is the purpose of this note to show that for any $n \geq 1$ the subset $F$ of $\mathbb{T}^{n}$ of all $x$ such that for any semisimple surjective endomorphism $\rho$ of $\mathbb{T}^{n}$ the closure of the orbit $\left\{\rho^{i}(x) \mid i=0,1,2, \ldots\right\}$ does not contain any element of finite order (in particular the orbit is not dense in $\mathbb{T}^{n}$ ) is still a large set in the sense that for any nonempty open set $\Omega$ of $\mathbb{T}^{n}, F \cap \Omega$ has Hausdorff dimension $n$ (cf. Corollary 2.7). In particular, $F$ is uncountable.

The proof involves showing that $F$ is a winning set of the Schmidt game (cf. $\$ 1$ ) for suitable values of the constants in the game. This is achieved using a result on winning sets in $\mathbb{R}^{n}$, proved by the present author in [1].

\section{Preliminaries}

In this we recall various notions and results involved in proving the main theorem.

\subsection{Endomorphisms of tori}

Let $\mathbb{T}^{n}$ be the $n$-dimensional torus where $n \geq 1$. We identify $\mathbb{T}^{n}$ as $\mathbb{R}^{n} / \mathbb{Z}^{n}$. In particular it is considered to be equipped with the metric given by

$$
\bar{d}\left(u+\mathbb{Z}^{n}, v+\mathbb{Z}^{n}\right)=\inf _{m \in \mathbb{Z}^{n}} d(u+m, v) \quad \text { for all } u, v \in \mathbb{R}^{n},
$$

where $d$ is the usual euclidean distance function on $\mathbb{R}^{n}$.

We denote by $e_{1}, \ldots, e_{n}$ the standard basis of $\mathbb{R}^{n}$; via the basis any $A \in G L(n, \mathbb{R})$ corresponds to a linear transformation of $\mathbb{R}^{n}$ which is also denoted by $A$. Any 
$A \in G L(n, \mathbb{Q}) \cap M(n, \mathbb{Z})$, namely a matrix with integer entries and nonzero determinant, yields a continuous surjective endomorphism $\bar{A}$ of $\mathbb{T}^{n}$ defined by

$$
\bar{A}\left(u+\mathbb{Z}^{n}\right)=A u+\mathbb{Z}^{n} \quad \text { for all } u \in \mathbb{R}^{n} .
$$

Conversely for any continuous surjective endomorphism $\rho$ of $\mathbb{T}^{n}$ there exists a unique $R \in G L(n, \mathbb{Q}) \cap M(n, \mathbb{Z})$ such that $\rho=\vec{R}(\mathrm{cf}$. [6]). $\bar{R}$ is said to be semisimple if $R$ is semisimple.

For a (continuous) surjective endomorphism $\rho$ of $\mathbb{J}^{n}$ and $x \in \mathbb{J}^{n}$ we denote by $C_{\rho}(x)$ the closure of the orbit of $x$, namely

$$
C_{\rho}(x)=\left\{\rho^{i}(x) \mid i=0,1,2, \ldots\right\}^{-} \text {. }
$$

\subsection{The Schmidt game}

In [4] W. M. Schmidt introduced a game which has been used in showing various sets to be large in a certain sense (cf. [2] and the references therein). The game involves two players say $\mathscr{A}$ and $\mathscr{B}$, two numbers $\alpha, \beta \in(0,1)$ corresponding to $\mathscr{A}$ and $\mathscr{B}$ respectively, and a complete metric space $X$. A sample procedure goes as follows: $\mathscr{R}$ starts the game by picking a closed ball $B_{0}$ in $X$ with arbitrary positive radius. Then $\mathscr{A}$ picks a closed ball $A_{1}$ contained in $B_{0}$ whose radius is $\alpha$ times that of $B_{0}$. Next $\mathscr{B}$ chooses a closed ball $B_{1}$ contained in $A_{1}$ having radius $\beta$ times that of $A_{1}$ and so on; inductively, after $\mathscr{B}$ has chosen $B_{k-1}, \mathscr{A}$ chooses a closed ball $A_{k}$ contained in $B_{k-1}$ with radius $\alpha$ times that of $B_{k-1}$ and $\mathscr{B}$ follows by choosing $B_{k}$ to be a closed ball contained in $A_{k}$ having radius $\beta$ times that of $A_{k}$. Since $X$ is a complete metric space, for each such sample procedure there is a unique point of intersection of the sets $B_{0} \supset A_{1} \supset B_{1} \supset A_{2} \supset B_{2} \cdots$. A subset $S$ of $X$ is said to be an $(\alpha, \beta)$-winning set (for $\mathscr{A}$ ) if there is a strategy by which $\mathscr{A}$ can ensure (by the choices during his turns) that, irrespective of how $\mathscr{B}$ makes the choices during his turns, the point of intersection belongs to $S$. A subset $S$ is said to be an $\alpha$-winning set if it is an $(\alpha, \beta)$-winning set for all $\beta \in(0,1)$.

Evidently the whole space $X$ is an $(\alpha, \beta)$-winning set for all $\alpha, \beta \in(0,1)$. It turns out that if $\beta \leq 2-\alpha^{-1}$, or equivalently $1-2 \alpha+\alpha \beta \leq 0$, then $X$ is the only $(\alpha, \beta)$ winning set (cf. [4] Lemma 5). On the other hand if $\beta>2-\alpha^{-1}$ then there can exist various proper subsets which are $(\alpha, \beta)$-winning sets and even $\alpha$-winning sets for $\alpha \in\left(0, \frac{1}{2}\right)$ (cf. Theorem 1.3 below).

Intuitively one would expect that $(\alpha, \beta)$-winning sets should be 'large'. We first note that this does not happen to be true in the 'conventional' sense; for instance, there exist subsets of $\mathbb{R}^{n}$ which are of first category and zero Lebesgue measure which are $(\alpha, \beta)$-winning sets for all $\alpha, \beta \in(0,1)$ such that $1-2 \alpha+\alpha \beta>0$. (cf. [4]). Nevertheless, thanks to the following results of Schmidt [4] the expectation is fulfilled in a certain sense.

THEOREM 1.1. If $S$ is an $(\alpha, \beta)$-winning subset of $\mathbb{R}^{n}$, with respect to the usual euclidean metric, then for any nonempty open set $\Omega \subseteq \mathbb{R}^{n}, S \cap \Omega$ has Hausdorf dimension at least

$$
\left(\log c_{n} \beta^{-n}\right) /|\log \alpha \beta|,
$$

where $c_{n}$ is a constant depending only on $n$. In particular, if $T$ is an $\alpha$-winning set then it is of Hausdorff dimension $n$. 
THEOREM 1.2. If $\left\{S_{k}\right\}_{1}^{\infty}$ is a sequence of $\alpha$-winning sets for some $\alpha \in(0,1)$ then $\bigcap_{1}^{\infty} S_{k}$ is also an $\alpha$-winning set.

It also turns out that for various classes of maps the images of winning sets are also winning sets for suitably modified values of the constants. This allows one to conclude that the winning sets are large by various stronger criteria. We refer to [5] and [2] for details concerning this theme.

\subsection{Winning sets in $\mathbb{R}^{n}$}

We now note a result which on the one hand provides a large class of examples of $(\alpha, \beta)$-winning sets in $\mathbb{R}^{n}$ and on the other hand provides a way of showing certain sets to be $(\alpha, \beta)$-winning; the latter aspect is relevant in $\S 2$.

We consider $\mathbb{R}^{n}$ equipped with the usual euclidean metric denoted by $d$. For any subset $E$ of $\mathbb{R}^{n}$ and $u \in \mathbb{R}^{n}$ we denote by $d(u, E)$ the distance of $u$ from $E$, namely the infemum of $\{d(u, v) \mid v \in E\}$. Similarly, for two subsets $E_{1}, E_{2}, d\left(E_{1}, E_{2}\right)$ denotes the distance between $E_{1}$ and $E_{2}$. For any subset $S$ of $\mathbb{R}^{n}$ the thickness of $S$ is defined to be

$$
\tau(S)=\inf _{W} \sup _{u, v \in S} d(u-v, W)
$$

where the infemum is taken over all hyperplanes $W$ of $\mathbb{R}^{n}$. The result in question is the following

THEOREM 1.3. Let $\alpha, \beta \in(0,1)$ be such that $1-2 \alpha+\alpha \beta>0$. Let $h \in \mathbb{N}$ be such that $(\alpha \beta)^{h}<\frac{1}{2}(1-2 \alpha+\alpha \beta)$ and let $\mu=(\alpha \beta)^{h}$. Let $P$ be a countable set and let $\{S(p, t)\}$ be a family of subsets of $\mathbb{R}^{n}$ (doubly) indexed by $p \in P$ and $t \in(0,1)$. Suppose that for any compact subset $C$ of $\mathbb{R}^{n}$ there exist $M \geq 1, \varepsilon \in(0,1)$ and a family $\left\{\tau_{p}\right\}_{p \in P}$ of positive numbers such that the following conditions are satisfied:

(a) if $p \in P$ and $t \in(0, \varepsilon)$ are such that $S(p, t) \cap C$ is nonempty then $\tau_{p} \leq M$ and $\tau(S(p, t)) \leq t \tau_{p}$.

(b) if $p, p^{\prime} \in P$ and $t \in(0, \varepsilon)$ are such that $S(p, t) \cap C$ and $S\left(p^{\prime}, t\right) \cap C$ are nonempty and $\mu \tau_{p} \leq \tau_{p^{\prime}} \leq \mu^{-1} \tau_{p}$ then either $p^{\prime}=p$ or $d\left(S(p, t), S\left(p^{\prime}, t\right)\right) \geq \varepsilon\left(\tau_{p}+\tau_{p^{\prime}}\right)$.

Then the set

$$
F=\bigcup_{\delta>0}\left(\mathbb{R}^{n}-\bigcup_{p \in P} S(p, \delta)\right)
$$

is an $(\alpha, \beta)$-winning set.

This is essentially Theorem 3.2 of [1], except that there we had demanded condition (b) for all $\mu \in(0,1)$ and correspondingly derived the conclusion for all $\alpha, \beta \in(0,1)$ such that $1-2 \alpha+\alpha \beta>0$. For a given pair $(\alpha, \beta)$ satisfying that condition, the value for $\mu$ was chosen precisely as in the statement of the above theorem: Thus the proof of Theorem 3.2 of [1] also yields the above theorem.

We refer the reader to [1] for examples of families satisfying the conditions of the theorem. 


\section{Results}

We now apply Theorem 1.3 to the study of orbits under endomorphism of tori following the notation as in $\S 1$. We begin with the following theorem.

THEOREM 2.1. Let $\rho$ be a semisimple surjective endomorphism of $\mathbb{T}^{n}$, where $n \geq 1$, and let $F_{\rho}=\left\{x \in \mathbb{T}^{n} \mid 1 \in \mathbb{T}^{n}-C_{\rho}(x)\right\}$, where 1 is the identity element in $\mathbb{T}^{n}$ (namely the image of $\mathbb{Z}^{n}$ in $\left.\mathbb{R}^{n} / \mathbb{Z}^{n}\right)$. Then $F_{\rho}$ is an $(\alpha, \beta)$-winning set for all $\alpha, \beta \in(0,1)$ such that $1-2 \alpha+\alpha \beta>0$. In particular it is an $\alpha$-winning set for all $\alpha$ such that $0<\alpha \leq \frac{1}{2}$.

Proof. Let $R \in G L(n, \mathbb{Q}) \cap M(n, \mathbb{Z})$ be the matrix inducing $\rho$. If some root of unity is an eigenvalue of $R$ then there exists a nontrivial character $\chi$ on $\mathbb{T}^{n}$ and $k \in \mathbb{N}$ such that the function $f=\chi+\chi \circ \rho+\cdots+\chi \circ \rho^{k-1}$ is $\rho$-invariant (cf. [6]). In this case $F_{\rho}$ contains the set $\left\{x \in \mathbb{T}^{n} \mid f(x) \neq f(1)\right\}$, which is readily seen to be the complement of a proper closed subgroup of $\mathbb{J}^{n}$. It is easy to see that such a subset is an ( $\alpha, \beta)$-winning set for all $\alpha, \beta$ such that $1-2 \alpha+\alpha \beta>0$. (See Lemma 3.7 in [1] for an idea of the proof.) We may therefore assume that no eigenvalue of $R$ is a root of unity. It is well known that for an integral matrix if all eigenvalues are of absolute value at most 1 then they are all roots of unity. Hence it follows from the assumption that $R$ has an eigenvalue of absolute value greater than 1 .

Now for $t>0$ let $B_{t}$ denote the open ball in $\mathbb{R}^{n}$ with center at 0 and radius $t$. Let

$$
F_{R}=\bigcup_{\delta>0}\left(\mathbb{R}^{n}-\bigcup_{j=0}^{\infty} R^{-j}\left(\mathbb{Z}^{n}+B_{\delta}\right)\right) .
$$

Then $F_{\rho}=F_{R} / \mathbb{Z}^{n}$. We also note that if $x \in \mathbb{T}^{n}$ and $u \in \mathbb{R}^{n}$ are such that $x=u+\mathbb{Z}^{n}$ then the closed ball in $\mathbb{T}^{n}$ with center at $x$ and radius $r>0$ is the image of the closed ball in $\mathbb{R}^{n}$ with center at $u$ and radius $r$. Therefore to prove the theorem it is enough to prove that $F_{R}$ is an $(\alpha, \beta)$-winning set for all $\alpha, \beta$ as in the hypothesis. We shall deduce this from Theorem 1.3 as follows.

Let $\alpha, \beta \in(0,1)$ such that $1-2 \alpha+\alpha \beta>0$ be fixed. Let $\mu=(\alpha \beta)^{h}$, where $h \in \mathbb{N}$ is such that $(\alpha \beta)^{h}<\frac{1}{2}(1-2 \alpha+\alpha \beta)$. Let $\lambda$ be the maximum of the absolute values of the eigenvalues of $R$; then $\lambda>1$ by our assumption. Let $l \in \mathbb{N}$ be such that $\lambda^{-l}<\mu$. Let $a=\mid$ det $\left.R\right|^{-1}$; then $R^{-j}\left(\mathbb{Z}^{n}\right) \subseteq a \mathbb{Z}^{n}$ for $1 \leq j \leq l$. Also let $b>0$ be such that $R^{-j}\left(B_{1}\right) \subseteq B_{b}$ for all $j=0,1, \ldots, l$. Then for any $k \in \mathbb{Z}^{+}$, viz. a nonnegative integer, and $t>0$ we have

$$
\bigcup_{j=k l}^{(k+1) l-1} R^{-j}\left(\mathbb{Z}^{n}+B_{\imath}\right) \subseteq a R^{-k l}\left(\mathbb{Z}^{n}\right)+R^{-k l}\left(B_{b t}\right)=R^{-k l}\left(a \mathbb{Z}^{n}+B_{b t}\right) .
$$

Now let $P=\mathbb{Z}^{n} \times \mathbb{Z}^{+}$and for any $p=(m, k) \in P$, where $m \in \mathbb{Z}^{n}$ and $k \in \mathbb{Z}^{+}$, and $t>0$ let

$$
S(p, t)=R^{-k l}\left(a m+B_{t}\right) .
$$

If $F$ is the set as in the conclusion of Theorem 1.3 for the family $\{S(p, t)\}$ as above, then it is easy to see that in view of (1) $F$ is contained in $F_{R}$. Therefore to prove the theorem it is enough to prove that the sets $\{S(p, t)\}$ satisfy the conditions of Theorem 1.3.

Let $V$ and $W$ be the largest $R$-invariant subspaces such that the absolute values of the eigenvalues of $R \mid V$ and $R \mid W$ are, respectively, equal to and less than $\lambda$. 
Then $\mathbb{R}^{n}=V+W$. Let $\pi \mid \mathbb{R}^{n} \rightarrow V$ be the corresponding projection onto $V$. Choosing hyperplanes containing $W$ in applying the definition we get that for any set $E$ in $\mathbb{R}^{n}$

$$
\tau(E) \leq \sup \{\|v\| \mid v \in \pi(E)\} .
$$

Since all eigenvalues of $R \mid V$ are of absolute value $\lambda$ and $R$ is semisimple there exists a constant $c>0$ such that

$$
\left\|R^{-j} v\right\| \leq c \lambda^{-j}\|v\| \quad \text { for all } v \in V \text { and } j \in \mathbb{Z}^{+} .
$$

Also let

$$
M=\sup \left\{\|v\| \mid v \in \pi\left(B_{1}\right)\right\}
$$

Then for any $p=(m, k) \in P$ and $t>0$ we have

$$
\begin{aligned}
\tau(S(p, t)) & =\tau\left(R^{-k l}\left(B_{t}\right)\right) \leq \sup \left\{\|v\| \mid v \in \pi\left(R^{-k l}\left(B_{t}\right)\right)\right\} \\
& =\sup \left\{\left\|R^{-k l} v\right\| \mid v \in \pi\left(B_{t}\right)\right\} \\
& \leq c M t \lambda^{-k l}
\end{aligned}
$$

Thus condition (a) of Theorem 1.3 holds if we choose

$$
\tau_{p}=c M \lambda^{-k l} \text { for } p=(m, k) \in P .
$$

(The condition holds independently of the compact set to be given). Now let $p=(m, k)$ and $p^{\prime}=\left(m^{\prime}, k^{\prime}\right)$ in $P$ be such that $\mu \tau_{p} \leq \tau_{p^{\prime}} \leq \mu^{-1} \tau_{p}$. Substituting from (2 and using the fact that $\lambda^{-l}<\mu$ (by the choice of $l$ ) we conclude that $k^{\prime}=k$. Now, for any $t>0$ we have

$$
\begin{aligned}
d\left(S(p, t), S\left(p^{\prime}, t\right)\right) & =d\left(R^{-k l}\left(a m+B_{t}\right), R^{-k l}\left(a m^{\prime}+B_{t}\right)\right) \\
& =d\left(a R^{-k l}\left(m^{\prime}-m\right), R^{-k l}\left(B_{2 t}\right)\right) .
\end{aligned}
$$

Since all eigenvalues of $R^{-1}$ are of absolute value at least $\lambda^{-1}$ and $R$ is semisimple there exists a constant $\sigma>0$ such that

$$
\left\|R^{-i} u\right\| \geq \sigma \lambda^{-i}\|u\| \text { for all } u \in \mathbb{R}^{n} \text { and } i \in \mathbb{Z}^{+} .
$$

Now let $s \in(0, a / 2)$ and

$$
\varepsilon=\min \{\sigma(a-2 s) / 2 c M, s\} .
$$

Then for any $m \in \mathbb{Z}^{n}-(0)$ and $k \in \mathbb{Z}^{+}$we have

$$
\begin{aligned}
d\left(a R^{-k l} m, R^{-k l}\left(B_{2 \varepsilon}\right)\right) & \geq d\left(\mathbb{R}^{n}-R^{-k l}\left(B_{a}\right), R^{-k l}\left(B_{2 \varepsilon}\right)\right) \\
& =d\left(\mathbb{R}^{n}-R^{-k l}\left(B_{a-2 \varepsilon}\right), 0\right),
\end{aligned}
$$

where $a-2 \varepsilon \geq a-2 s>0$. In view of (4) the right hand side is at least $\sigma(a-2 \varepsilon) \lambda^{-k l}$. Also by the choice of $\varepsilon$ we have $\sigma(a-2 \varepsilon) \lambda^{-k l} \geq \sigma(a-2 s) \lambda^{-k l} \geq 2 c M \varepsilon \lambda^{-k l}=$ $\varepsilon\left(\tau_{p}+\tau_{p^{\prime}}\right)$, by (2). In view of (3) and (5) this shows that condition (b) of Theorem 1.3 is also satisfied, for $\varepsilon$ as above, and hence by that theorem $F_{R}$ is an $(\alpha, \beta)$-winning set. This completes the proof of the theorem.

Corollary 2.7. Let $\mathbb{T}^{n}=\mathbb{R}^{n} / \mathbb{Z}^{n}, n \geq 1$, and let

$$
Q=\mathbb{Q}^{n} / \mathbb{Z}^{n}=\left\{z \in \mathbb{T}^{n} \mid z^{r}=1 \text { for some } r \in \mathbb{N}\right\} \text {. }
$$

Let $F$ be the set of all $x$ in $\mathbb{T}^{n}$ such that for any semisimple surjective endomorphism $\rho$ of $\mathbb{T}^{n}, Q \cap C_{\rho}(x)=\varnothing$ (i.e., the closure of $\left\{\rho^{i}(x) \mid i \in \mathbb{Z}^{+}\right\}$is disjoint from $Q$ ). Then 
$F$ is an $\alpha$-winning set for all $\alpha$ such that $0<\alpha \leq \frac{1}{2}$. Further, for any nonempty open subset $\Omega$ of $\mathbb{T}^{n}, F \cap \Omega$ is of Hausdorff dimension $n$. In particular $F$ is uncountable.

Proof. Let $0<\alpha \leq \frac{1}{2}, \rho$ an endomorphism as in the hypothesis and $z \in Q$. There exists a finite $\rho$-invariant subgroup $C$ of $\mathbb{T}^{n}$ containing $z$. Applying Theorem 2.1 to the factor endomorphism $\bar{\rho}$ on $\mathbb{T}^{n} / C$, which is also an $n$-dimensional torus, we deduce that the set

$$
\left\{x \in \mathbb{T}^{n} \mid z \text { is not contained } C_{\rho}(x)\right\}
$$

is an $\alpha$-winning set. Since $Q$ is countable, applying Theorem 1.2 we can conclude that

$$
\left\{x \in \mathbb{T}^{n} \mid Q \cap C_{\rho}(x)=\varnothing\right\}
$$

is an $\alpha$-winning set. Again since there are only countably many endomorphisms the same theorem yields that the set $F$ in the statement of the corollary is an $\alpha$-winning set. Now let $\tilde{F}=\left\{u \in \mathbb{R}^{n} \mid u+\mathbb{Z}^{n} \in F\right\}$. then clearly $\tilde{F}$ is an $\alpha$-winning set in $\mathbb{R}^{n}$. Hence by Theorem 1.1 for any nonempty open subset $\Omega$ of $\mathbb{R}^{n}, \tilde{F} \cap \Omega$ has Hausdorff dimension $n$. This readily implies that $F \cap \Omega$ is of Hausdorff dimension $n$ for all nonempty open subsets $\Omega$ of $\mathbb{T}^{n}$.

\section{Miscellaneous comments and questions}

(1) If $\rho$ is a semisimple automorphism of $\mathbb{J}^{n}$ then by Corollary $2.7 F_{\rho} \cap F_{\rho-1}$ is an $\alpha$-winning for all $\alpha \in\left(\begin{array}{ll}0 & \frac{1}{2}\end{array}\right]$; that is, the set of $\chi \in \mathbb{T}^{n}$ such that $Q \cap\left\{\rho^{i}(x) \mid i \in \mathbb{Z}\right\}^{-}=$ $\varnothing$ is an $\alpha$-winning set for all $\alpha \in\left(\begin{array}{ll}0 & \frac{1}{2}\end{array}\right]$. In particular it is incompressible in the sense of [2]. This result was announced in [2] for $n=2$. There we raised the question whether a similar assertion holds more generally for all Anosov diffeomorphisms.

(2) The method of proof of Theorem 2.1 can also be applied to obtain a similar result for certain commutative semigroups of surjective endomorphisms and an analogue of the above for commutative groups of automorphisms of $\pi^{n}, n \geq 2$. It may be noted however that for the action of the group of all automorphisms and also for certain (non-commutative) subgroups the situation is quite the opposite, in that the orbit of any $x \notin Q$ is dense in $\pi^{n}$.

(3) If $R \in G L(n, \mathbb{Z})$ is hyperbolic (that is, no eigenvalue of $R$ has an absolute value 1) and irreducible over $\mathbb{Q}$ and $\rho$ is the automorphism of $\mathbb{T}^{n}$ induced by $R$ then by a result of $\mathrm{R}$. Mane [3] any rectifiable path in $\mathbb{J}^{n}$ contains a point $x$ whose orbit under $\rho$ is dense in $\mathbb{T}^{n}$. Thus it follows that for such a $\rho$ the set $F_{\rho}$ does not contain any rectifiable path in $\mathbb{T}^{n}$. What about other automorphisms? Are these sets connected?

\section{REFERENCES}

[1] S. G. Dani. Bounded orbits of flows on homogeneous spaces. Comment. Math. Helv. 61 (1986), 636-660.

[2] S. G. Dani. On badly approximable numbers, Schmidt games and bounded orbits of flows. Preprint. 
[3] R. Mane. Orbits of paths under hyperbolic toral automorphisms. Proc. Amer. Math. Soc. 73 (1979), 121-125.

[4] W. M. Schmidt. On badly approximable numbers and certain games. Trans. Amer. Math. Soc. 123 (1966), 178-199.

[5] W. M. Schmidt. Diophantine Approximation, Springer-Verlag: Berlin-Heidelberg-New York, 1980.

[6] P. Walters. An Introduction to Ergodic Theory, Springer-Verlag: Berlin-Heidelberg-New York, 1982. 